\title{
Editorial: Recent Advances in Thermally Activated Delayed Fluorescence Materials
}

\author{
Chihaya Adachi ${ }^{1}$, Guohua Xie ${ }^{2}$, Sebastian Reineke ${ }^{3}$ and Eli Zysman-Colman ${ }^{4 *}$ \\ ${ }^{1}$ Center for Organic Photonics and Electronics Research (OPERA), Kyushu University, Fukuoka, Japan, ${ }^{2}$ Hubei Key \\ Laboratory on Organic and Polymeric Optoelectronic Materials, Department of Chemistry, Sauvage Center for Molecular \\ Sciences, Wuhan University, Wuhan, China, ${ }^{3}$ Dresden Integrated Center for Applied Physics and Photonic Materials (IAPP), \\ School of Science, Technische Universität Dresden, Dresden, Germany, ${ }^{4}$ Organic Semiconductor Centre, EaStCHEM School \\ of Chemistry, University of St Andrews, St. Andrews, United Kingdom
}

Keywords: thermally activated delay fluorescence (TADF), OLED - organic light emitting devices/display, organic semiconductor (OSC), metal complexes, polymers

\section{Editorial on the Research Topic}

\section{Recent Advances in Thermally Activated Delayed Fluorescence Materials}

Thermally activated delayed fluorescence (TADF) materials design has rapidly evolved over the past 8 years since Adachi and co-workers demonstrated that organic donor-acceptor compounds could recruit both singlet and triplet excitons in electroluminescent devices, reaching nearly $100 \%$ internal quantum efficiency (Uoyama et al., 2012). Accompanying new and improved TADF materials has been the enhanced performance of the organic light-emitting diodes (OLEDs), underpinned by a refined understanding of the TADF mechanism and the design rules that govern materials design.

This themed issue of Frontiers in Chemistry documents recent advances in TADF materials. Paisley et al. reviewed stimuli-responsive TADF in polymer nanoparticles and how these are relevant to sensing and imaging. Yin et al. provided an overview of TADF polymer materials. Etherington summarized the solid-state solvation effects and aggregate effects in organic TADF emitters. Barman et al. reviewed mechanochromic organic TADF materials. Lee et al. focused on boron containing TADF materials. To et al. contributed a perspective on metal complexes that showed TADF and their use as emitters in OLEDs. Hamze et al. demonstrated highly efficient deep blue luminescence of 2-coordinate coinage metal complexes. Crucho et al. reported on TADF dye-loaded nanoparticles for fluorescence live-cell imaging. Li Y. et al. showed how through-space charge transfer states can be recruited within polymers to produce high-performance solutionprocessed OLEDs, while Chen et al. showed how similar excited states can be exploited in small molecule TADF emitters. Mubarok et al. likewise exploited through-space CT states in triarylborane-containing TADF emitters. Kusakabe et al. revealed the importance of the relative orientation of donor and acceptor groups in through space TADF small molecule emitters. TADF polymer design was featured in the work of Yang et al. who reported white OLEDs while red OLEDs were reported by Zhan et al.. Franco et al. demonstrated how TADF absorption can be sensitized by using variable length oligo(phenylene ethynylene) antennae. Cai et al. revealed how aggregationinduced delayed fluorescence materials can be recruited for high-performance solution-processed OLEDs. Li H. et al. provided examples of small molecule emitters that showed aggregationinduced delayed fluorescence. Distinct high triplet energy ambipolar TADF host materials were independently reported by Rodella et al. for vacuum-deposited OLEDs and Godumala et al. for solution-processed OLEDs. High-performance OLEDs also require horizontally oriented emitters. 
Naqvi et al. revealed molecular design rules for small molecular TADF emitters to exhibit more preferentially horizontal orientation. Sasabe et al. demonstrated how molecular orientation could be controlled in carbazole-containing host materials. Improved performances of blue TADF OLEDs have been of particular focus within the OLED community. Zhang et al. reported on a family of blue emitters containing an OBOFused Benzo[fg]tetracene acceptor. Sohn et al. demonstrated high-performance blue OLEDs using a pyrimidine-containing TADF emitter. Tsuchiya et al. showed how computational modeling can effectively guide blue emitter design. The greater exploration of chemical space has led to unorthodox emitter design. For instance, delayed fluorescence was reported by Pandey et al. in compounds containing dianthrylboron-based donor-acceptor systems, while Krotkus et al. showed fast delayed fluorescence in pyridazine-based emitters.

\section{REFERENCES}

Uoyama, H., Goushi, K., Shizu, K., Nomura, H., and Adachi, C. (2012). Highly efficient organic light-emitting diodes from delayed fluorescence. Nature 492, 234-238. doi: 10.1038/nature11687

Conflict of Interest: The authors declare that the research was conducted in the absence of any commercial or financial relationships that could be construed as a potential conflict of interest.
The articles in this themed issue provide insight into how TADF materials design has evolved and how the use of TADF materials has expanded beyond OLEDs to sensing and imaging. Despite the intense focus over the past 8 year, this collection clearly demonstrates that there is still much to learn and much from which to be inspired.

\section{AUTHOR CONTRIBUTIONS}

All authors listed have made a substantial, direct and intellectual contribution to the work, and approved it for publication.

\section{FUNDING}

EZ-C thanks EPSRC (EP/P010482/1) for financial support.
Copyright (C) 2020 Adachi, Xie, Reineke and Zysman-Colman. This is an open-access article distributed under the terms of the Creative Commons Attribution License (CC $B Y)$. The use, distribution or reproduction in other forums is permitted, provided the original author(s) and the copyright owner(s) are credited and that the original publication in this journal is cited, in accordance with accepted academic practice. No use, distribution or reproduction is permitted which does not comply with these terms. 